\title{
Influence of Agroclimatic Indices on Morphological and Growth Attributes of Maize (Zea mays L.)
}

\author{
M. Swetha Sree*, V. Raja Rajeswari, T. Prathima, P. Latha and P. Sudhakar \\ Department of Crop Physiology, S.V Agricultural College, Tirupati-517502, \\ Andhra Pradesh, India \\ *Corresponding author
}

\section{A B S T R A C T}

Keywords

Morphology,

Growth, GDD,

HTU, PTU and

HUE

Article Info

Accepted:

20 May 2018

Available Online:

10 June 2018
A field experiment was conducted during kharif season, 2016 at S.V. Agricultural College, Tirupathi to study the 'Influence of Agro-climatic indices on morphological and growth attributes of maize (Zea mays L.)'. The experiment was laid out in split plot design with twelve treatments. Three maize hybrids D.S 900M, Pinnacle and CP818 with four dates of sowing (June II FN, July I FN, July II FN and August I FN). The results indicated that the number of days to attain different phenological growth stages varied. The morphological attributes and growth attributes like plant height, Leaf area, LAI were highest for Pinnacle and June I sowing $\left(D_{1}\right)$. Among the hybrids higher leaf $\left(15.6 \mathrm{~g}\right.$ plant $\left.{ }^{-1}\right)$, stem $(78.9 \mathrm{~g}$ plant $\left.{ }^{1}\right)$, tassel $\left(2.6 \mathrm{~g} \mathrm{plant}^{-1}\right)$ and cob weight $\left(177 \mathrm{~g} \mathrm{plant}^{-1}\right)$ was observed in D.S 900M on par with Pinnacle and CP818. Among the dates of sowing significantly higher leaf $(21.6 \mathrm{~g}$ plant $\left.{ }^{-1}\right)$, stem $\left(95.9 \mathrm{~g}\right.$ plant $\left.^{-1}\right)$, tassel $\left(3.2 \mathrm{~g} \mathrm{plant}^{-1}\right)$ and cob weight $\left(192 \mathrm{~g} \mathrm{plant}^{-1}\right)$ was observed in $\mathrm{D}_{1}$ (June II FN). Variabilty in morphological and growth attributes tested maize hybrids at different dates of sowing is attributed to variability in crop requirement of temperature and photoperiod viz., Growing degree days (GDD), Photothermal Units (PTU) and Heliothermal units (HTU) especially at grand growth phase stage of maize.

\section{Introduction}

A range of climatic factors affect the crop growth and development. Temperature is the most important among all environmental factors that influences rate of plant growth. Grain yield of corn differs considerably across the growing areas even when soil fertility and moisture supply are near optimal. Reasons for such variation might be differences in sun shine hours or temperature etc. Temperature influences both timing and duration of the each phenophase. Excess of radiation and high temperatures are often the most limiting factors affecting plant growth and final crop yield. High temperatures can cause considerable pre- and post-harvest damages, including scorching of leaves and twigs, sunburns on leaves, branches and stems, leaf senescence and abscission, shoot and root growth inhibition, fruit discoloration and damage, and reduced yield. High-temperatureinduced modifications in plants may be direct as on existing physiological processes or indirect in altering the pattern of development. These responses may differ from one 
phenological stage to another. Heat stress, singly or in combination with drought, is a common constraint during anthesis and grain filling stages in many cereal crops. The grain filling phase is the most important factor in explaining the reduced yields at warmer temperatures (White and Reynolds, 2003).

\section{Materials and Methods}

The experiment was conducted during kharif season, 2016 at S.V. Agricultural College, Tirupati located at $13.65^{\circ} \mathrm{N}$ and $79.42^{\circ} \mathrm{E}$, which is situated in southern agro climatic region of Andhra Pradesh. The experiment was laid out in split plot design with twelve treatments and three replications. The experiment was raised with hybrids as major treatments and dates of sowing as subtreatments. The maize hybrids D.S 900M, Pinnacle and CP818 were sown at four dates of sowing (June II FN, July I FN, July II FN and August I FN). The dates of occurance of different phenological events viz., emergence, six leaf stage, tasselling stage, silking stage, soft dough stage, hard dough stage and physiological maturity stage. Plant height (cm), Leaf area, Leaf area index, plant dry weights was estimated in three plants in each plot at phenological growth stages of crop

The daily meteorological data from the regional meteorological office situated $1 \mathrm{~km}$ away from the experimental field, were used. The agro meteorological indices growing degree days (GDD), Photothermal unit (PTU), Heliothermal Unit (HTU) and Heat Use Efficiency (HUE) were calculated using following formulae of Rajput (1980):

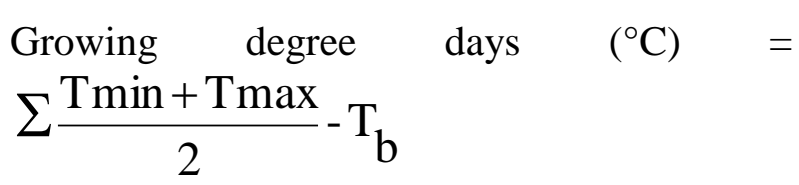

Accumulated PTU $\left({ }^{\circ} \mathrm{C}\right.$ day $\left.\mathrm{hr}\right)=\mathrm{GDD} \times$ Day length (hrs.)
Accumulated HTU $\left({ }^{\circ} \mathrm{C}\right.$ day $\left.\mathrm{hr}\right)=\mathrm{GDD} \times$ Duration of sunshine hours

$\operatorname{HUE}\left(\mathrm{kg} \mathrm{ha}^{-1 \circ} \mathrm{C} \mathrm{day}^{-1}\right)=\frac{\text { Dry w tof grains / unit area }}{\text { GDD }}$

Here,

Tmin= Minimum temperature

Tmax $=$ Maximum temperature

$\mathrm{T}_{\mathrm{b}}=$ Base temperature $=8{ }^{\circ} \mathrm{C}$

\section{Results and Discussion}

\section{Phenology}

The number of days to attain physiological maturity increased with delay in sowing. Among the hybrids Pinnacle recorded highest number of days taken to physiological maturity (106) followed by D.S900M (104) and CP818 (102). Their was no significant variability among all the growth stages of maize hybrids except at tasseling stage where CP818 recorded more number of days to attain anthesis stage. Among the dates of sowing $\mathrm{D}_{4}$ (August I FN) recorded more number of days to attain anthesis and maturity days $(61,113)$ followed by $\mathrm{D}_{3}, \mathrm{D}_{1}$ and $\mathrm{D}_{2}$. Delayed sowings extended life cycle of maize crop under these southern Agroclimatic Zone. Similar variability was also observed by Amgain et al., (2011) and Majumder et al., (2016).

\section{Growing Degree Days}

During the crop growing season, GDD accumulated across all the sowing dates ranged from 3170.4 to $2920.8^{\circ} \mathrm{C}$ and hybrids 2994.1to $3053.3^{\circ} \mathrm{C}$ from sowing to physiological maturity. The GDD accumulation was higher in case of Pinnacle followed by D.S $900 \mathrm{M}$ and CP818 from sowing to physiological maturity. The shifting of sowing dates corresponds to fluctuations in temperatures either lengthing or shortening of the growing periods. $\mathrm{D}_{1}$ (June II FN) recorded 
highest GDD followed by $\mathrm{D}_{2}$ and $\mathrm{D}_{3}$. GDD varied among all the crop growth stages due to the fluctuated unfavourable conditions high temperatures during the crop growth period. The accumulated heat units decreased for different phenological growth stages as the sowings delayed. Similar variability was also reported by Pandey et al., (2010), Girijesh et al., (2011) and Ahmad et al., (2016)

\section{Photothermal Unit}

During the crop growing season, Mean PTU across all the hybrids and sowing dates was $35903.1^{\circ} \mathrm{C}$ day hour and $37023.2^{\circ} \mathrm{C}$ day hour respectively. The PTU was higher in case of Pinnacle $\left(36151.1^{\circ} \mathrm{C}\right.$ day hour) followed by D.S $900 \mathrm{M}$ and CP818 from sowing to physiological maturity. $\mathrm{D}_{1}$ (June II FN) recorded highest PTU $\left(38837.4^{\circ} \mathrm{C}\right.$ day hour) followed by $\mathrm{D}_{2}$ and $\mathrm{D}_{3}$. Due to increased number of calendar days to attain physiological maturity $\mathrm{D}_{4}$ recorded higher PTU $\left(3677.04^{\circ} \mathrm{C}\right.$ day hour). Similar variability was also observed by Gowda et al., (2013).

\section{Heliothermal Units (HTU)}

During the crop growing season, HTU across all the hybrids and sowing dates was $16980.88^{\circ} \mathrm{C}$ day hour and $18811.8^{\circ} \mathrm{C}$ day hour respectively. The HTU was higher in case of Pinnacle $\left(17098^{\circ} \mathrm{C}\right.$ day hour) followed D.S 900Mand CP818 from sowing to physiological maturity. $\mathrm{D}_{1}$ (June $\mathrm{II} \quad \mathrm{FN}$ ) recorded highest $\mathrm{HTU}\left(20288^{\circ} \mathrm{C}\right.$ day hour) and $\mathrm{D}_{4}$ recorded lowest HTU $\left(14163.4^{\circ} \mathrm{C}\right.$ day hour). Similar variability was also observed by Girijesh et al., (2011) and Hariram et al., (2012).

\section{Heat Use Efficiency}

During the crop growing season, Mean HUE across all the hybrids and sowing dates was 1.0and 1.0. The HUE was higher in case of
Pinnacle and $\mathrm{CP} 818$ was $1 \mathrm{~kg} \mathrm{ha}^{-1}{ }^{\mathrm{o}} \mathrm{C} \mathrm{day}^{-1}$ and D.S $900 \mathrm{M}\left(0.91 \mathrm{~kg} \mathrm{ha}^{-1}{ }^{\mathrm{o}} \mathrm{C} \mathrm{day}^{-1}\right)$ from sowing to physiological maturity. Among the dates of sowing $\mathrm{D}_{1}$ (June II FN) recorded highest HUE $\left(1.2 \mathrm{~kg} \mathrm{ha}^{-1}{ }^{\circ} \mathrm{C}\right.$ day $\left.^{-1}\right)$ and lowest by $\mathrm{D}_{4}$ (August I FN, $0.6 \mathrm{~kg} \mathrm{ha}^{-1}{ }^{\circ} \mathrm{C} \mathrm{day}^{-1}$ ).

Higher HUE represents that plant utilized the heat more efficiently by increasing biological activity and higher grain yield. Similar variability was also observed by Thavaprakash et al., (2007), Girijesh et al., (2011) and Rajesh et al., (2015) in wheat.

\section{Plant Height (cm)}

Among the three hybrids Pinnacle recorded significantly highest plant height at silking stage $(165 \mathrm{~cm})$ followed by D.S $900 \mathrm{M}(158.8$ $\mathrm{cm})$ whereas CP818 recorded lowest plant height $(146.7 \mathrm{~cm})$.

Among the dates of sowing significant variability was seen throughout the growth stages among the four dates of sowing. D1 (June II FN) recorded significantly highest plant height $(165 \mathrm{~cm})$ at all growth stages followed by D2 (July I FN), D3 (July II FN) and D4 (August I FN). Variability in plant height of tested maize hybrids at different dates of sowing is attributed to variability in crop requirement of temperature and photoperiod viz, Growing degree days (GDD), Photothermal Units (PTU) and Heliothermal units (HTU). Similar variability among the was also reported by Williams and Lindsquist (2007) and Hussain et al., (2012).

\section{Leaf dry weight $\left(\mathrm{g} \mathrm{plant}^{-1}\right)$}

Among the hybrids highest leaf dry weight was recorded by D.S $900 \mathrm{M}$ (24.7 $\left.\mathrm{g} \mathrm{plant}^{-1}\right)$ due to higher accumulation of GDD, HTU and PTU followed by Pinnacle (22.5 g plant $\left.{ }^{-1}\right)$ and lowest leaf dry weight was recorded in CP818 (21.4 $\left.\mathrm{g} \mathrm{plant}^{-1}\right)$. 
Table.1 Number of days to attain different phenophases of maize as influenced by different dates of sowing

\begin{tabular}{|c|c|c|c|c|c|c|c|}
\hline Treatments & Emergence & $\begin{array}{l}6 \text { leaf } \\
\text { stage }\end{array}$ & $\begin{array}{l}\text { Tasseling } \\
\text { stage }\end{array}$ & $\begin{array}{l}\text { Silking } \\
\text { stage }\end{array}$ & $\begin{array}{l}\text { Soft } \\
\text { dough } \\
\text { stage }\end{array}$ & $\begin{array}{l}\text { Hard } \\
\text { dough } \\
\text { stage }\end{array}$ & $\begin{array}{l}\text { Physiological } \\
\text { maturity }\end{array}$ \\
\hline \multicolumn{8}{|l|}{ Hybrids } \\
\hline $\mathrm{H}_{1}:$ D.S 900M & 6 & 24 & 58 & 67 & 77 & 88 & 105 \\
\hline$\overline{\mathrm{H}_{2}}$ : Pinnacle & 7 & 25 & 58 & 67 & 80 & 96 & 106 \\
\hline $\mathrm{H}_{3}: \mathrm{CP818}$ & 6 & 24 & 61 & 69 & 79 & 90 & 102 \\
\hline $\mathrm{CD}(\mathrm{P}=0.05)$ & NS & NS & 0.1 & NS & NS & NS & NS \\
\hline \multicolumn{8}{|l|}{ Dates of Sowing } \\
\hline $\begin{array}{l}D_{1}: \text { June II } \\
\text { FN }\end{array}$ & 5 & 23 & 57 & 67 & 76 & 92 & 104 \\
\hline $\mathrm{D}_{2}$ : July I FN & 5 & 22 & 56 & 63 & 72 & 84 & 100 \\
\hline $\mathrm{D}_{3}:$ July II FN & 4 & 24 & 60 & 67 & 78 & 88 & 100 \\
\hline $\begin{array}{l}\mathrm{D}_{4}: \text { August I } \\
\text { FN }\end{array}$ & 7 & 25 & 61 & 69 & 83 & 94 & 113 \\
\hline $\mathrm{CD}(\mathrm{P}=0.05)$ & 0.2 & 0.2 & 0.1 & NS & NS & NS & NS \\
\hline
\end{tabular}

Table.2 Calendar days and agrometeorological indices at different growth stages of maize hybrids at different dates of sowing

\begin{tabular}{|c|c|c|c|c|c|}
\hline \multirow[t]{2}{*}{ Treatments } & \multicolumn{5}{|c|}{ Sowing to Physiological maturity } \\
\hline & Cday & GDD & PTU & HTU & $\begin{array}{l}\text { HUE } \\
\text { at maturity }\end{array}$ \\
\hline \multicolumn{6}{|l|}{ Hybrids } \\
\hline $\mathrm{H}_{1}$ : D.S 900M & 104 & 3049.1 & 36101.1 & 17074 & 0.9 \\
\hline $\mathrm{H}_{2}$ : Pinnacle & 112 & 3053.3 & 36151.1 & 17098 & 1.0 \\
\hline H. : CP818 & 102 & 2994.7 & 35457.2 & 16770.3 & 1.0 \\
\hline Mean & 106 & 3032.3 & 35903.1 & 16980.8 & 1.0 \\
\hline SD & 5.3 & 32.7 & 387.0 & 183.1 & 0.0 \\
\hline $\mathrm{CV}(\%)$ & 5.0 & 1.1 & 1.1 & 6.2 & 4.8 \\
\hline \multicolumn{6}{|l|}{ Dates of sowing } \\
\hline$D_{1}$ : June II FN & 104 & 3170.4 & 38837.4 & 20288 & 1.2 \\
\hline $\mathrm{D}_{2}$ : July I FN & 100 & 2990 & 36358.4 & 19734 & 1.0 \\
\hline $\mathrm{D}_{\mathbf{3}}$ : July II FN & 100 & 2920.8 & 34932.8 & 15501.9 & 0.9 \\
\hline $\mathrm{H}_{4}$ : August I FN & 112 & 3079.6 & 3677.0 & 14163.4 & 0.6 \\
\hline Mean & 103 & 3065.2 & 37023.2 & 18811.8 & 1 \\
\hline SD & 6.9 & 129.9 & 1731.0 & 2222.3 & 0.3 \\
\hline CV (\%) & 6.7 & 4.2 & 4.6 & 11.8 & 28.4 \\
\hline
\end{tabular}


Table.3 Effect temperature on leaf dry weight $\left(\mathrm{g} \mathrm{plant}^{-1}\right)$ of maize hybrids sown at different dates of sowing

\begin{tabular}{|c|c|c|c|c|c|c|}
\hline Treatments & $\begin{array}{l}6 \text { leaf } \\
\text { stage }\end{array}$ & $\begin{array}{c}\text { Tasseling } \\
\text { stage }\end{array}$ & $\begin{array}{l}\text { Silking } \\
\text { stage }\end{array}$ & $\begin{array}{l}\text { Soft dough } \\
\text { stage }\end{array}$ & $\begin{array}{c}\text { Hard } \\
\text { dough } \\
\text { stage }\end{array}$ & $\begin{array}{c}\text { Physiological } \\
\text { maturity }\end{array}$ \\
\hline \multicolumn{7}{|l|}{ Hybrids } \\
\hline $\mathrm{H}_{1}:$ D.S 900M & 6.4 & 15.9 & 24.7 & 18.1 & 15.3 & 15.6 \\
\hline $\mathrm{H}_{2}$ : Pinnacle & 5.8 & 15.4 & 22.5 & 15.2 & 13.1 & 11.9 \\
\hline$H_{3}: C P 818$ & 5.6 & 14.6 & 21.4 & 15.8 & 15.2 & 12.2 \\
\hline $\mathrm{CD}(\mathrm{P}=0.05)$ & Ns & 0.6 & 1.4 & 1.4 & 0.8 & 0.7 \\
\hline \multicolumn{7}{|l|}{ Dates of Sowing } \\
\hline D+ $+_{1}:$ June II FN & 12.4 & 19.5 & 28.9 & 24.4 & 23.4 & 21.6 \\
\hline $\mathrm{D}_{2}$ : July I FN & 7.0 & 17.2 & 24.8 & 15.5 & 13.5 & 12.6 \\
\hline $\mathrm{D}_{3}$ : July II FN & 2.5 & 13.9 & 22.7 & 13.7 & 11.5 & 10.1 \\
\hline $\mathrm{D}_{4}:$ August I FN & 1.6 & 10.6 & 14.9 & 11.8 & 10.3 & 8.8 \\
\hline $\mathrm{CD}(\mathrm{P}=0.05)$ & 0.6 & 1.3 & 2.7 & 1.6 & 1.4 & 1.2 \\
\hline
\end{tabular}

Table.4 Effect of temperature on stem dry weight $\left(\mathrm{g} \mathrm{plant}^{-1}\right)$ of maize hybrids sown at different dates of sowing

\begin{tabular}{|c|c|c|c|c|c|c|}
\hline Treatments & $\begin{array}{l}6 \text { leaf } \\
\text { stage }\end{array}$ & $\begin{array}{l}\text { Tasseling } \\
\text { stage }\end{array}$ & $\begin{array}{l}\text { Silking } \\
\text { stage }\end{array}$ & $\begin{array}{l}\text { Soft dough } \\
\text { stage }\end{array}$ & $\begin{array}{c}\text { Hard } \\
\text { dough } \\
\text { stage }\end{array}$ & $\begin{array}{l}\text { Physiological } \\
\text { maturity }\end{array}$ \\
\hline \multicolumn{7}{|l|}{ Hybrids } \\
\hline $\mathrm{H}_{1}$ : D.S 900M & 5.5 & 57.6 & 77.3 & 77.6 & 78.6 & 78.9 \\
\hline $\mathrm{H}_{2}$ : Pinnacle & 5.4 & 53.4 & 74.7 & 76.4 & 77.0 & 77.5 \\
\hline $\mathrm{H}_{3}: \mathrm{CP} 818$ & 5.3 & 47.9 & 75.1 & 75.7 & 76.3 & 76.5 \\
\hline $\mathrm{CD}(\mathrm{P}=0.05)$ & NS & 2.7 & NS & NS & NS & NS \\
\hline \multicolumn{7}{|l|}{ Dates of Sowing } \\
\hline $\mathrm{D}_{1}$ : June II FN & 9.3 & 85.2 & 93.9 & 94.8 & 95.2 & 95.9 \\
\hline $\mathrm{D}_{2}$ : July I FN & 7.5 & 66.8 & 81.8 & 82.6 & 83.2 & 83.6 \\
\hline$D_{3}:$ July II FN & 2.4 & 35.6 & 69.4 & 70.3 & 71.3 & 71.7 \\
\hline $\mathrm{D}_{4}:$ August I FN & 2.3 & 24.4 & 57.6 & 58.6 & 59.5 & 59.6 \\
\hline $\mathrm{CD}(\mathrm{P}=0.05)$ & 0.8 & 5.2 & 4.6 & 4.2 & 2.9 & 3.1 \\
\hline
\end{tabular}

Table.5 Effect of temperature on root dry weight $\left(\mathrm{g}_{\text {plant }}{ }^{-1}\right)$ of maize hybrids sown at different dates of sowing

\begin{tabular}{|c|c|c|c|c|c|c|}
\hline Treatments & $\begin{array}{l}6 \text { leaf } \\
\text { stage }\end{array}$ & $\begin{array}{l}\text { Tasseling } \\
\text { stage }\end{array}$ & $\begin{array}{l}\text { Silking } \\
\text { stage }\end{array}$ & $\begin{array}{c}\text { Soft } \\
\text { dough } \\
\text { stage }\end{array}$ & $\begin{array}{l}\text { Hard dough } \\
\text { stage }\end{array}$ & $\begin{array}{c}\text { Physiological } \\
\text { maturity }\end{array}$ \\
\hline \multicolumn{7}{|l|}{ Hybrids } \\
\hline $\mathrm{H}_{1}$ : D.S 900M & 6.24 & 14.7 & 25.4 & 29.4 & 28.0 & 25.3 \\
\hline $\mathrm{H}_{2}$ : Pinnacle & 6.4 & 15.6 & 22.9 & 28.4 & 27.5 & 26.2 \\
\hline $\mathrm{H}_{3}: \mathrm{CP} 818$ & 6.4 & 14.6 & 22.5 & 27.7 & 25.3 & 24.3 \\
\hline $\mathrm{CD}(\mathrm{P}=0.05)$ & NS & NS & 1.4 & NS & 1.7 & 0.8 \\
\hline \multicolumn{7}{|l|}{ Dates of Sowing } \\
\hline $\mathrm{D}_{1}$ : June II FN & 13.2 & 26.8 & 26.7 & 42.7 & 42.5 & 41.0 \\
\hline $\mathrm{D}_{2}$ : July I FN & 8.3 & 15.3 & 28.2 & 27.4 & 24.8 & 21.6 \\
\hline
\end{tabular}


Int.J.Curr.Microbiol.App.Sci (2018) 7(6): 2582-2590

\begin{tabular}{|c|c|c|c|c|c|c|}
\hline $\mathrm{D}_{3}$ : July II FN & 2.3 & 10.6 & 20.8 & 22.6 & 21.2 & 19.4 \\
\hline $\mathrm{D}_{4}:$ August I FN & 1.6 & 7.1 & 18.6 & 21.3 & 19.5 & 19.0 \\
\hline $\mathrm{CD}(\mathrm{P}=0.05)$ & 0.5 & 1.0 & 1.5 & 1.2 & 3.3 & 1.7 \\
\hline
\end{tabular}

Table.6 Effect of temperature on tassel dry weight $\left(\mathrm{g}_{\text {plant }}{ }^{-1}\right)$ of maize hybrids sown at different dates of sowing

\begin{tabular}{|c|c|c|c|c|c|}
\hline Treatments & Tasseling stage & $\begin{array}{c}\text { Silking } \\
\text { stage }\end{array}$ & $\begin{array}{c}\text { Soft dough } \\
\text { stage }\end{array}$ & $\begin{array}{l}\text { Hard dough } \\
\text { stage }\end{array}$ & $\begin{array}{l}\text { Physiological } \\
\text { maturity }\end{array}$ \\
\hline \multicolumn{6}{|l|}{ Hybrids } \\
\hline $\mathrm{H}_{1}:$ D.S 900M & 4.9 & 4.2 & 3.8 & 3.2 & 2.6 \\
\hline $\mathrm{H}_{2}$ : Pinnacle & 4.9 & 4.1 & 3.9 & 3.2 & 2.5 \\
\hline$H_{3}: C P 818$ & 4.5 & 4.1 & 3.7 & 3.1 & 2.3 \\
\hline $\mathrm{CD}(\mathrm{P}=0.05)$ & 0.2 & 0.2 & 0.1 & 0.1 & 0.1 \\
\hline \multicolumn{6}{|l|}{ Dates of Sowing } \\
\hline $\mathrm{D}_{1}$ : June II FN & 5.4 & 4.7 & 4.2 & 3.9 & 3.1 \\
\hline $\mathrm{D}_{2}:$ July I FN & 5.1 & 4.3 & 3.9 & 3.2 & 2.7 \\
\hline $\mathrm{D}_{3}:$ July II FN & 4.5 & 4.1 & 3.7 & 2.8 & 2.2 \\
\hline $\mathrm{D}_{4}:$ August I FN & 3.9 & 3.5 & 3.3 & 2.7 & 1.8 \\
\hline $\mathrm{CD}(\mathrm{P}=0.05)$ & 0.3 & 0.1 & 0.2 & 0.1 & 0.2 \\
\hline
\end{tabular}

Table.7 Effect of temperature on cob dry weight $\left(\mathrm{g} \mathrm{plant}^{-1}\right)$ of maize hybrids sown at different dates of sowing

\begin{tabular}{|c|c|c|c|c|}
\hline Treatments & Silking stage & Soft dough stage & Hard dough stage & $\begin{array}{c}\text { Physiological } \\
\text { maturity }\end{array}$ \\
\hline \multicolumn{5}{|l|}{ Hybrids } \\
\hline $\mathrm{H}_{1}:$ : D.S 900M & 18.5 & 52.7 & 145.7 & 177 \\
\hline $\mathrm{H}_{2}$ : Pinnacle & 18.0 & 51.9 & 144.5 & 181.2 \\
\hline $\mathrm{H}_{3}: \mathrm{CP} 818$ & 16.9 & 50.7 & 139.8 & 175.2 \\
\hline $\mathrm{CD}(\mathrm{P}=0.05)$ & 0.7 & 0.7 & 2.0 & 2.3 \\
\hline \multicolumn{5}{|l|}{ Dates of Sowing } \\
\hline $\mathrm{D}_{1}$ : June II FN & 21.5 & 58.5 & 157.5 & 193.2 \\
\hline $\mathrm{D}_{2}:$ July I FN & 19.2 & 55.7 & 153.5 & 183.4 \\
\hline $\mathrm{D}_{3}$ : July II FN & 16.9 & 48.1 & 135.4 & 172 \\
\hline $\mathrm{D}_{4}:$ August I FN & 13.8 & 44.7 & 127 & 162.5 \\
\hline $\mathrm{CD}(\mathrm{P}=0.05)$ & 0.9 & 1.2 & 1.5 & 3.1 \\
\hline
\end{tabular}

Table.8 Effect of temperature on leaf area $\left(\mathrm{cm}^{2}\right)$ of maize hybrids sown at different dates of sowing

\begin{tabular}{|c|c|c|c|c|c|c|}
\hline Treatments & $\begin{array}{l}6 \text { leaf } \\
\text { stage }\end{array}$ & $\begin{array}{l}\text { Tasseling } \\
\text { stage }\end{array}$ & $\begin{array}{l}\text { Silking } \\
\text { stage }\end{array}$ & $\begin{array}{c}\text { Soft } \\
\text { dough } \\
\text { stage }\end{array}$ & $\begin{array}{l}\text { Hard dough } \\
\text { stage }\end{array}$ & $\begin{array}{l}\text { Physiological } \\
\text { maturity }\end{array}$ \\
\hline \multicolumn{7}{|l|}{ Hybrids } \\
\hline $\mathrm{H}_{1}$ : D.S 900M & 501.4 & 2666.4 & 2766.7 & 1929.3 & 1305.9 & 51.8 \\
\hline $\mathrm{H}_{2}$ : Pinnacle & 467.7 & 2582.1 & 2911.1 & 1998.1 & 1350.3 & 49.1 \\
\hline $\mathrm{H}_{3}: \mathrm{CP818}$ & 493.3 & 2462.3 & 2847.3 & 1876.7 & 1201.8 & 47.9 \\
\hline $\mathrm{CD}(\mathrm{P}=0.05)$ & NS & NS & NS & NS & 77.5 & 1.6 \\
\hline \multicolumn{7}{|l|}{ Dates of Sowing } \\
\hline $\mathrm{D}_{1}$ : June II FN & 831.3 & 4495.7 & 4532.1 & 2976.9 & 2460.7 & 62.2 \\
\hline
\end{tabular}




\begin{tabular}{|l|c|c|c|c|c|c|}
\hline $\mathbf{D}_{2}$ : July I FN & 501.4 & 2537.7 & 2876.2 & 2096.1 & 1222.7 & 51.7 \\
\hline $\mathbf{D}_{3}$ : July II FN & 356.0 & 1870.4 & 1937.1 & 1417.3 & 850.5 & 49.2 \\
\hline $\mathbf{D}_{4}$ : August I FN & 261.0 & 1380.2 & 1541.2 & 1249.7 & 610.3 & 35.3 \\
\hline $\mathrm{CD}(\mathbf{P}=\mathbf{0 . 0 5})$ & 176.5 & 180.5 & 812.2 & $\mathrm{NS}$ & 152 & 2.3 \\
\hline
\end{tabular}

Table.9 Effect of temperature on leaf area index (LAI) of maize hybrids sown at different dates of sowing

\begin{tabular}{|c|c|c|c|c|c|c|}
\hline Treatments & $\begin{array}{l}6 \text { leaf } \\
\text { stage }\end{array}$ & $\begin{array}{l}\text { Tasseling } \\
\text { stage }\end{array}$ & $\begin{array}{l}\text { Silking } \\
\text { stage }\end{array}$ & $\begin{array}{l}\text { Soft dough } \\
\text { stage }\end{array}$ & $\begin{array}{c}\text { Hard } \\
\text { dough } \\
\text { stage }\end{array}$ & $\begin{array}{l}\text { Physiological } \\
\text { maturity }\end{array}$ \\
\hline \multicolumn{7}{|l|}{ Hybrids } \\
\hline $\mathrm{H}_{1}$ : D.S 900M & 0.42 & 2.3 & 2.2 & 1.6 & 1.1 & 0.043 \\
\hline $\mathrm{H}_{2}$ : Pinnacle & 0.39 & 2.4 & 2.8 & 1.7 & 1.2 & 0.041 \\
\hline $\mathrm{H}_{3}: \mathrm{CP818}$ & 0.41 & 2.1 & 2.1 & 1.6 & 1 & 0.04 \\
\hline $\mathrm{CD}(\mathrm{P}=0.05)$ & NS & NS & 0.12 & NS & 0.06 & 0.001 \\
\hline \multicolumn{7}{|l|}{ Dates of Sowing } \\
\hline $\mathrm{D}_{1}:$ June II FN & 0.69 & 3.7 & 3.8 & 2.5 & 2.1 & 0.052 \\
\hline $\mathrm{D}_{2}$ : July I FN & 0.42 & 2.3 & 2.2 & 1.7 & 1.0 & 0.042 \\
\hline $\mathrm{D}_{3}:$ July II FN & 0.29 & 1.6 & 1.6 & 1.1 & 0.7 & 0.041 \\
\hline $\mathrm{D}_{4}:$ August I FN & 0.22 & 1.2 & 1.1 & 1.0 & 0.5 & 0.029 \\
\hline $\mathrm{CD}(P=0.05)$ & 0.14 & 0.44 & 0.25 & 0.074 & 0.12 & 0.002 \\
\hline
\end{tabular}

Among the dates of sowing D1 (June II FN) recorded significantly highest leaf dry weight at silking stage (28.9 $\mathrm{g} \mathrm{plant}^{-1}$ ) followed by D2, D3 and D4.

The plant leaf dry weight decreased as the sowings delayed. Lowest plant leaf dry weight (12.5 g plant $^{-1}$ ) was recorded in the D4 (August I FN).

\section{Stem dry weight (g plant $\left.{ }^{-1}\right)$}

Among the hybrids D. S 900 M (78.9 g plant $^{-}$ $\left.{ }^{1}\right)$ stem dry weight was numerically higher than Pinnacle (77.47 $\left.\mathrm{g} \mathrm{plant}^{-1}\right)$ and CP818 $\left(76.50 \mathrm{~g}\right.$ plant $\left.^{-1}\right)$. Significant difference among the hybrids was found only at tasseling stage.

Among the dates of sowing D1 (June II Fortnight) recorded significantly higher stem dry weight (95.9 g plant $^{-1}$ ) compared to all the other dates of sowing (D2, D3 and D4) at all the growth stages of crop growth. Higher stem dry weight at first date of sowing can be correlated to higher accumulation of GDD and PTU from sowing to Tasseling stage. Similar variability among the interaction hybrids and dates of sowing in maize was also reported by Nielsen et al., (2002) and Sulochana et al., (2015).

\section{Root dry weight (g plant-1)}

Among the hybrids D.S $900 \mathrm{M}$ recorded numerically higher root dry weight $(29.4 \mathrm{~g}$ plant $^{-1}$ ) at soft dough stage followed by Pinnacle (28.4 g plant $\left.^{-1}\right)$ and lowest root dry weight was recorded by CP818.

Among the dates of sowing D1 (June II FN) recorded significantly higher root dry weight (42.7 g plant $^{-1}$ ) among all growth stages of crop growth followed by D2, D3 and D4 recorded significantly lower root dry weight (21.3 g plant $\left.^{-1}\right)$. Similar variability among the interaction hybrids and dates of sowing in maize was also reported by Nielsen et al., (2002) and Sulochana et al., (2015).

Tassel Dry Weight (g plant $\left.{ }^{-1}\right)$

Among the hybrids D.S 900M (H1) and 
Pinnacle (H2) recorded significantly higher tassel dry weight ( $4.9 \mathrm{~g}$ plant $\left.^{-1}\right)$ compared to CP818 (4.5 $\left.\mathrm{g} \mathrm{plant}^{-1}\right)$.

Among the dates of sowing D1 (June II FN) recorded significantly higher tassel dry weight $\left(5.4 \mathrm{~g} \mathrm{plant}^{-1}\right)$ among all growth stages of crop growth followed by D2, D3 and D4 recorded significantly lower tassel dry weight $(3.9 \mathrm{~g}$ plant $^{-1}$ ) Similar variability among the interaction hybrids and dates of sowing in maize was also reported by Nielsen et al., (2002) and Sulochana et al., (2015).

\section{Cob Dry Weight $\left(\mathrm{g} \mathrm{plant}^{-1}\right)$}

Among the hybrids D.S 900M (H1) and Pinnacle (H2) recorded significantly higher cob dry weight (179.8 $\mathrm{g}$ plant $\left.^{-1}\right)$ compared to CP818 (4.5 $\mathrm{g}$ plant $\left.^{-1}\right)$. Among the dates of sowing D1 (June II FN) recorded significantly higher cob dry weight (194 g plant ${ }^{-1}$ ) among all growth stages of crop growth followed by D2, D3 and D4 recorded significantly lower tassel dry weight $\left(162.5 \mathrm{~g} \mathrm{plant}^{-1}\right)$.

Variability in the total dry matter of tested maize hybrids at different dates of sowing is attributed to variability in crop requirement of temperature and photoperiod viz., Growing degree days (GDD), Photothermal Units (PTU) and Heliothermal units (HTU).Similar variability among the interaction hybrids and dates of sowing in maize was also reported by Nielsen et al., (2002) and Sulochana et al., (2015).

\section{Leaf area $\left(\mathrm{cm}^{2}\right.$ plant $\left.^{-1}\right)$}

Among the hybrids maximum leaf area was recorded at silking stage by Pinnacle (2911.1 $\mathrm{cm}^{2}$ plant $\left.^{-1}\right)$ followed by CP $818\left(2847 \mathrm{~cm}^{2}\right.$ plant $^{-1}$ ). Lowest leaf area was recorded by D.S 900 M (2766.65 $\mathrm{cm}^{2}$ plant $\left.{ }^{-1}\right)$. Radiation interception largely determines the leaf area index. The results are in conformity with the findings of Muchow and Carberry (1989), Sulochana et al., (2015) in maize crop.

Among the dates of sowing D1 (June II FN) recorded significantly higher leaf area (4532 $\mathrm{cm}^{2}$ plant ${ }^{-1}$ ) at all the growth stages except at soft dough stage. D1 (June second fortnight) was significantly higher than D2 (July I FN), D3 (July II FN), D4 (August I FN) at all the growth stages except at soft dough stage. Higher GDD and PTU accumulation at six leaf stage to tasseling stage during June II FN resulted in higher leaf area because of higher daily average temperatures compared to D2, D3 and D4.The results are in conformity with the findings of Muchow and Carberry (1989) and Zaker et al., (2014).

\section{Leaf Area Index (LAI)}

Among the hybrids maximum LAI was recorded by Pinnacle (2.8) followed by D.S 900M (2.2) and CP818 (2.1). Increase in LAI for Pinnacle can be explained as it accumulated higher GDD and PTU compared compared to other hybrids. Among the dates of sowing D1 (June II FN) recorded significantly highest LAI (3.8) followed by D2 (July I FN, 2.2), D3 (July II FN, 1.6) and lowest by D4 (August I FN, 1.1) at all the growth stages of maize. The results are in confirmity with the finding of Zaker et al., (2014).

\section{References}

Amgain, L.P. Accumulated heat unit and phenology of diverse maize varieties as effected by planting dates under Rampur conditions, Nepal. Agronomy Journal of Nepal. 2: 201-205 (2011)

Gowda, T.P., Halikatti, S.I. and Manjunatha, S.B. Thermal requirement of maize (Zea mays L.) as influenced by planting dates and cropping systems. Research Journal of Agricultural Sciences. 4(2): 207-210 (2013). 
Hariram, Gurjot, S., Mavi, G.S and Sohu, V.S. Accumulated heat unit requirement and yield of irrigated wheat (Triticum aestivum L.) varieties under different crop growing environment incentral Punjab. Journal of Agrometeorology. 14(2): 147153 (2012).

Hossein, M.H and Masoud, M. Effect of the plant density and sowing date on kernel yield in early maturing maize. International journal of plant, animal and environmental sciences. 4- 4 (2014).

Hussain, M., Shabir, G., Farooq, M., Jabran, K and Farooq, S. 2012. Developmental and phenological responses of wheat to sowing dates. Pakistan Journal of Agricultural Science. 49(4): 459-468

Majumder, D., Kingra, P.K and Kukal, S.S. Canopy temperature and heat use efficiency of spring maize under modified soil microenvironment. Annual J ournal of gricultural Research. 37(3): 225-235 (2016).

Majumder, D., Kingra, P.K and Kukal, S.S. Canopy temperature and heat use efficiency of spring maize under modified soil microenvironment. Annual J ournal of agricultural Research. 37(3): 225-235 (2016).

Muchow, R.C. 1989. Comparative productivity of maize, sorghum and pearmillet in a semi-arid tropical environment. 1: Yield potential. Field Crops Research. 20(3): 191-205.

Nielsen, R.L., Tomison, P.R., Brown, G.A., Holter, A.L., Wells, J. and Wuethrick, K. L. 2002. Delayed planting effects on flowering and grain maturation of dent corn. Agronomy Journal. 94: 549-558.
Rajesh, K., Deshmukh, R., Verma, $\mathrm{N}$ and Kaushik, D. Phenological growth and yield of wheat in relation to agrometeorological indices under different sowing dates. Plant Archives. 15: 81-87 (2015).

Rajput, R.P. Response of soyabean crop to climate and soil environment $P h . D$ thesis. Indian Agricultural Research Institute, Pusa, New Delhi (1980).

Shrestha, U., Amgain, L.P., Bahadur, T.K., Dahal, K.R and Shrestha, J. 2016. Effect of sowing dates and maize cultivars in growth and yield of maize along with their Agro-climatic indices in Nawalprarasi.Nepal Journal of Agricultural Research. 3(1): 57-62.

Thavapraash, N., Jagannath, R., Velayudham, K and Guruswamy, L. Seasonal influence on phenology and accumulated heat units in relation to yield of baby corn. International Journal of Agricultural Research. 2(9): 826-831 (2007).

White, J.W. and Reynolds, M.P. 2003. A physiological perspective on modeling temperature response in wheat and maize crops. In: White JW (ed) Modeling temperature response in wheat and maize. Proceedings of a Workshop, CIMMYT, El Batán, Mexico, 23-25 April 2001. CIMMYT, Mexico City, p 8-17

Williams, M.M and Lindqiut, J.L. 2007. Influence of planting date and weed interference on sweet corn growth and development. Agronomy Jurnal. 99: 1066-1072.

Zaker, S., Aminpanah, H, Homayoon, D. 2014. Effect of planting date and nitrogen rate on yield and quality of forage corn. Agriculture and Forestry. 60(2): 193-206.

\section{How to cite this article:}

Swetha Sree M., V. Raja Rajeswari, T. Prathima, P. Latha and Sudhakar P. 2018. Influence of Agroclimatic Indices on Morphological and Growth Attributes of Maize (Zea mays L.). Int.J.Curr.Microbiol.App.Sci. 7(06): 2582-2590. doi: https://doi.org/10.20546/ijcmas.2018.706.305 